This item was submitted to Loughborough's Research Repository by the author.

Items in Figshare are protected by copyright, with all rights reserved, unless otherwise indicated.

\title{
The effect of barriers on the tidal range in estuaries
}

PLEASE CITE THE PUBLISHED VERSION

LICENCE

CC BY-NC-ND 4.0

\section{REPOSITORY RECORD}

Tang, Yong Ming. 2019. "The Effect of Barriers on the Tidal Range in Estuaries". figshare. https://hdl.handle.net/2134/452. 


\title{
The Effect of Barriers on The Tidal Range in Estuaries
}

\author{
Yong Ming Tang*
}

\begin{abstract}
Numerical simulations of the shallow water equations are used to study the effect of topographic barriers on the tidal range in estuaries. The results show that the nonlinear advection terms in the momentum equations play very little role, and that the interaction between the tide and the topographic barriers is mostly due to the bottom friction, which is represented by the usual quadratic Chezy law.
\end{abstract}

\footnotetext{
*Department of Mathematical Sciences, Loughborough University, LE11, 3TU, UK
} 


\section{Introduction}

The purpose of this paper is to describe the effect of topographic barriers on the tidal range in large estuaries, using numerical simulations of the shallow water equations. Because many regions experience large tides, there has been much interest in constructing tidal barrages, either to control the tide or to extract power. Correspondingly there have been several studies made of the effect of such tidal barrages on the flow region, using laboratory models, numerical simulations and in situ observations (see, for instance, Falconer and Yu (1991), and Riddle and Lewis (1998)). Here, however, we are more concerned with large-scale topographic effects, whether natural or constructed, although our results could be used, in extrapolation, to infer some properties of tidal barrages.

We consider topographic barriers, which either extend across the estuary below the ebb-tide level thus allowing the tidal flow to penetrate into the landward end of the estuary over the top of the barrier, or extend above the flood tide level, but only partially across the estuary thus allowing the tidal flow to penetrate into the landward end of the estuary around the side of the barrier through the gap between the barrier and the estuary land boundary (see Figure 1). Our concern is to determine which combination of barrier parameters and tidal forcing heights lead to the most effective blocking of the tidal flow, and consequently the greatest effect on the tidal range, particularly at the landward end of the estuary. There have been, of course, many studies of the tidal flow over and around barriers which have focussed on the flow dissipation at the barrier, and related issues (see, for instance the Proceedings of the Eighth International Conference on Hydraulic Engineering Software published as Hydraulic Engineering Software VIII, and similar recent volumes, for an overview of recent work in this area). But we need to emphasise that the details of the local behaviour at the barrier is not our main concern here; rather we are concerned with the larger-scale effect on the tidal range, while acknowledging that this is, of course, determined by what happens at the barrier.

In Section 2 we formulate the governing equation and system parameters, based on the familiar shallow water equations. It will emerge that the bottom stress term is crucial in the present simulations, and this is modelled in the usual way by a Chezy law, which is quadratic in the velocity field, with an empirically determined drag coefficient. But, in some cases when the flow was particularly strong and the nonlinear advective terms were also of some importance, we found it necessary to parametrise the loss of energy in the large-scale flow due to the generation of short-scale waves. This we do by modifying the drag coefficient in the Chezy law in a novel way; the details of this modification are described in the Appendix. Then in Section 3 we present our numerical results, and in Section 4 we discuss some implications of our results. 


\section{Formulation}

\section{Governing equations}

In standard notation, the two-dimensional barotropic shallow water equations are

$$
\begin{gathered}
u_{t}+u u_{x}+v u_{y}-f v+g \zeta_{x}=-\frac{\tau_{B}^{(x)}}{\rho H}, \\
v_{t}+u v_{x}+v v_{y}+f u+g \zeta_{y}=-\frac{\tau_{B}^{(y)}}{\rho H}, \\
\zeta_{t}+(H u)_{x}+(H v)_{y}=0 .
\end{gathered}
$$

where $H=h+\zeta, h(x, y)$ is the undisturbed water depth, $\zeta$ is the surface elevation, $(u, v)$ are the velocity components in the $(x, y)$ directions (see Figure 1$), f$ is the Coriolis

parameter, $g$ is gravitational acceleration, $\rho$ is water density, and $\tau_{B}^{(x, y)}$ are the frictional stress components.

At the closed boundaries (e.g. river banks) we need to ensure that there is no flow across the boundary. In the numerical discretisation of equations (2.1-3) we enforce this condition by imposing sufficiently large friction over the land region to reduce the velocity field there to zero. This has the advantage of allowing us to use a rectangular computational grid even when the boundary is irregular; in practice it is sufficient to use only a small number of land points. This device has been successfully used by us in several coastal ocean simulations (see, for instance Tang et al 1996, 1997), and has some similarity to the concept of sponge layers at the domain boundaries.

\section{Tidal forcing}

The tidal forcing is specified at the estuary mouth (i.e. the open boundary). Let $\zeta_{T}$ be the tidal component at the boundaries. For simplicity, in this study we input the semi-diurnal lunar constituent $\left(M_{2}\right)$ only.

$$
\zeta_{T}=\zeta_{0} \cos \left(\sigma t-\phi_{0}\right)
$$

Here $\zeta_{0}$ and $\phi_{0}$ are the amplitude and phase of the tidal constituent respectively. Because the ocean tide will typically have a very large length scale, it is sufficient for our purpose to assume that at the estuary mouth, $\zeta_{T}$ is a function of time only (as show in (2.6)) so that $\zeta_{0}$ and $\phi_{0}$ are constants. Without loss of generality, we set $\phi_{0}=0$, and vary $\zeta_{0}$ over the range 0.1 to 1 meter. To avoid high-frequency transients, we ramp up the tidal forcing with a linear variation of $\zeta_{0}$ in time, from 0 to its maximum final value, over a period of 6 hours.

\section{Frictional parametrization}

The usual Chezy law is used for bottom friction, so that the $\mathrm{x}$ - and $\mathrm{y}$-components of the bottom stress are given by,

$$
\tau_{B}^{(x, y)}=C_{D} q(u, v)
$$


where the flow speed $q$ is defined as $q=\sqrt{u^{2}+v^{2}}$, and we choose the drag coefficient to have the value $C_{D}=2.6 \times 10^{-3}$ (see, for instance, Csanady, 1984 or Gill, 1982). For purposes of comparison, we also consider the linear bottom friction law

$$
\tau_{B}^{(x, y)}=\alpha_{0}(u, v),
$$

where $\alpha_{0}=1.2 \times 10^{-1} \mathrm{~cm} / \mathrm{s}$ (e.g. Csanady, 1984).

Because the estuary has a relatively small size, and particularly when the tidal barrier is inserted, and the tidal forcing is quite strong, we found that our numerical simulations tended to produce short-scale temporal and spatial oscillations, sometimes leading to numerical instability. This phenomenon occured in conjunction with those cases when the nonlinearity was already quite strong, so that the nonlinear advective terms in the momentum equations were significant. Although these oscillations could extend down to the grid-scale and could consequently be interpreted as numerical artefacts, we nevertheless interpret their presence as evidence that the fluid flow, especially near the barrier, is attempting to produce short-scale waves, whose spatial scales are much less than those defined by the estuarine and barrier geometry, and whose temporal scales are much less than the forcing period. This is an expected physical effect, but in the context of the shallow water equations, these cannot be properly represented, and hence their possible generation provides an energy sink for the large-scale flow as modelled by the shallow water equations. This process is sometimes modelled by introducing a horizontal eddy viscosity coefficient in a traditional viscous term in the horizontal momentum equations. Another approach would treat the process in a conservative manner, and retain higherorder dispersive terms in the horizontal momentum equations, thus replacing equations (2.1-3) with a Boussinesq system. Both these options raise the order of the governing differential equations, with a consequent increase in the computational complexity of the numerical scheme.

Here, we follow a different approach, which parametrises these short-scale waves as an energy-loss from the large-scale flow, but does not increase the order of the governing equations. Our approach is outlined in the Appendix, and the outcome is that in the bottom friction law (2.5) the drag coefficient is enhanced, so that (2.5) is replaced with

$$
\tau_{B}^{(x, y)}=\left\{C_{D}+\alpha F^{3 / 2}\right\} q(u, v) .
$$

Here $F=q^{2} / g H$ is the square of the local Froude number, and $\alpha$ is an empirical constant whose value is set at 100 for reasons we shall describe shortly. In essence, we argue that, on dimensional grounds, the enhanced friction will scale proportionally to $q^{2}$, and that the associated energy loss will depend on the Froude number. An outline of the analysis leading to the $F^{3 / 2}$ law in (2.7) is given in the Appendix. To determine the empirical coefficient $\alpha$ we carried out several simulations, without the presence of a barrier, and varied $\alpha$ until short-scale oscillations of temporal scale much less than 1 hour, and a spatial scale of much less than $1 \mathrm{~km}$ were reduced to an order of magnitude less than $1 / 100$ of the large-scale flow. But we emphasise here that this enhancement of the frictional drag coeeficient was only needed and significant when the nonlinear advective terms were also significant, and for many of our results, that was not the case. 


\section{Estuary and Barrier geometry}

We consider two estuarine geometries, shown in Figure 1. The first, case (A) is a model of an estuary for which only the along-channel variations are significant. It has a uniform width $Y$ which is varied over the range 3.5 to $4.0 \mathrm{~km}$, a length $X$ which is varied over the range 20 to $27 \mathrm{~km}$, and is such that the depth $h$ varies linearly from the estuary mouth where it is equal to $30 \mathrm{~m}$, to a very small depth $(1.2 \mathrm{~m})$ at the inner land boundary.

The second, case (B) is a model of an estuary which has both along-channel and across-channel variations. It has a width $Y=3.0 \mathrm{~km}$ at the estuary mouth, but this width then decreases linearly along the channel (i.e in the negative $\mathrm{x}$-direction) to 1.6 $\mathrm{km}$ at the inner land end. In this case, the depth profile $h(x, y)$ is parabolic across the width of the estuary, being effectively zero at the side walls. Along the centre line of the estuary, the depth varies linearly, as in case (A).

In both cases (A) and (B), a topographic barrier is inserted, as shown in Figure 1 but with a zero horizontal gap (i.e. $G=0$ where $G$ denotes the horizontal gap at the location of the minimum depth of the barrier below the initial sea-level), and a finite vertical gap (i.e. $D>0$ where $D$ is the minimum depth of the barrier below the initial sea-level); thus the barrier extends across the channel but below the initial sea-level. In the along-channel direction (i.e $x$-direction) the barrier has a sinusoidal profile and has a total along-channel extent $E$; the barrier is uniform in the across-channel direction (i.e. $y$-direction). The barrier is centred at about 5 to $5.5 \mathrm{~km}$ from the estuary mouth (where the initial water depth is about 22 meters in the absence of the barrier). In our simulations we vary both the minimum depth $D$ of the barrier and its along-channel extent $E$.

Further, we consider a situation for case (A) only when the barrier leaves a finite, non-zero horizontal gap (i.e. $G>0$ ), but extends above the flood tide level (i.e. $D \leq 0$, so that there is no vertical gap (see Figure 1). In this case we again use the same alongchannel sinusoidal profile for the barrier with along-channel extent $E$. We call this case (C).

\section{Numerical results}

The equations of motion (2.1-3) are discretized as described in Tang and Grimshaw (1995, 1996). Briefly, the horizontal momentum equations (2.1) and (2.2) are integrated forward in time using a trapezoidal integration rule (IS THIS SO) to give the transport (i.e. $(h u, h v)$ at the time step $t+\Delta t$ in terms of the velocity field at time $t$ and the pressure gradient (i.e. $\left.\left(\zeta_{x}, \zeta_{y}\right)\right)$ split equally between the time levels. The nonlinear advection terms in the horizontal momentum equations are first written in a flux-conserving form, then discretised using Green's integral theorem, and evaluated at the time step $t$. The result is substituted into equation (2.3) for the conservation of mass, which is then stepped forward in time using an implicit Crank-Nicholson method. The land boundary conditions are incorporated into the momentum equations as described earlier. At the estuary mouth we impose the tidal forcing (2.4).

The parameter settings for the study domain are that the initial undisturbed water

depth at the estuary mouth is $h_{\max }=30 \mathrm{~m}$. Here the tidal forcing is applied with 
$\zeta_{0}=0.1,0.3$, or 1.0 meters. With no barrier present, the tidal height, at each instant of time, is almost unchanged along the length of the estuary, while the current field decreases from the estuary mouth to zero at the landward end. This is to be expected, as the natural length scale, namely the barotropic radius of deformation, associated with the tidal forcing frequency, is much greater than the estuary length $X$.

First we consider case(A) as described above. A barrier is placed at the location, where the initial undisturbed water depth is 22 meters. We carried out the simulations for a maximum barrier height of 20 and 17 meters, so that the vertical gap $D$ is 2 and 5 meters respectively, while the barrier width $E$ was 750 and 1500 meters respectively. The grid spacing $\Delta x$ in the numerical scheme is varied between 50 meters to 150 meters, as needed.

Figure 2 shows comparisons of the water level at the two points just before (solid line) and just after the barrier (dashed line) for different tidal inputs of $\zeta_{0}=0.1,0.3$, and 1.0 meters. The results show that the water levels are significantly lower at the point after the barrier. But more importantly the results show that the effect of the barrier is much stronger for the $\zeta_{0}=1.0$ meter input, than for an input of $\zeta_{0}=0.3$, and 0.1 meters, and this effect is not proportional to the level of inputs. Thus, for a weak tidal forcing input of $\zeta_{0}=0.1$ meters, the effect of the barrier is hardly discernible, but for a strong tidal forcing input of $\zeta_{0}=0.1$ meters, the barrier reduces the tide by an order of magnitude. We infer that the effect of the barrier on the tide is thus due to the nonlinear interaction between the tide and topographic barrier. To investigate this further, we repeated the calculations without the nonlinear advective terms on the left-hand side of the momentum equation, but significantly retained the nonlinear quadratic friction term. The results (Figure 3 ) show no substantial meaningful difference from Figure 2. This implies that the origin of this nonlinear interaction is due to the nonlinear friction term.

To test this from another angle, we we replaced the quadratic bottom friction with the linear friction law (2.6). Figure 4 shows the results one would expect from a linear theory, namely that the resuts scale with the input forcing amplitude, and so, in Figure 4, we can discern no substantial difference except that the peak amplitudes are reduced by a factor of 10 when the tidal forcing input is similarly reduced.

The conclusion from this sequence of simulations is clear. The interaction between the tidal wave and topographic barrier is due to the nonlinear friction law, acting at the barrier. The nonlinear advection terms in the momentum equations play an insignificant role in this phenomenon. We repeated this sequence of simulations for different parameter settings, and reached the same conclusion (the results are not shown here). Further, because the nonlinear advective are not significant for this effect, this result also does not depend significantly on the new friction coefficient $\alpha F^{3 / 2}$ we introduced in (2.7).

To study the effect of the barrier width $E$, and depth $D$ below the undisturbed sea-level, we reduced the resolution to $\Delta x=50$ meters, and carried out the following simulations with a parameter setting of a tidal forcing input $\zeta_{0}=0.5$ and 1.0 meters, a barrier width $E$ in the along-channel direction of of $300,400,500, \cdots 1300$ meters, and a height of $15,17,20$ meters; that is, the depth $D=7.0,5.0$ and 2.0 meters respectively below the undisturbed sea-level.

Grid sensitivity studies were carried out by varying $\Delta x$, and in particular we found 
that a smaller value did not change the main features of the results shown here, but, of course, increased the computational time for each simulation.

Figure 5a and 5b plot the ratio of the peak sea-level at a point (L) after the barrier, and a point $(\mathrm{S})$ before the barrier, as a function of the barrier width $E$ for different barrier depths $D$. The left-hand panel is for a tidal forcing input of $\zeta_{0}=1.0$ meter, while the right-hand panel is for $\zeta_{0}=0.5$ meters. For a barrier height of 20 meters (solid lines, the corresponding depth $D=2$ meters), the reduction of water level due to the barrier is markedly high. Further, this effect is more significant for an input of $\zeta_{0}=1.0$ meter than for $\zeta_{0}=0.5$ meters.

The same results for case (B) are shown in Figure 6, for a an estuary which narrows as well as becomin shallower. The trends are the same, but interestingly, the effect of the barrier per se is somewhat reduced.

In case $(\mathrm{C})$ we use the same estuarine gemeotry setting as case (A), but instead of looking into the results of varying the barrier depth $D$ and its width $E$, we fix the height of the barrier to be greater than the undisturbed sea-level (i.e. $D \leq 0$, see Figure 1) and the examine the effect of having a gap in the transverse direction, i.e. $G>0$. As in Figures 5 and 6 , in Figure 7 a we show the ratio of sea-level at a point $L$ after the barrier and a point $S$ before the barrier as a function of the non-dimensionalized barrier gap width (i.e. $G / Y$ where $\mathrm{Y}$ is the total fixed width of the estuary). The tidal forcing input $\zeta_{0}=1.0$ meters. In Figure $7 \mathrm{~b}$ we show the transports in the $x$ - and $y$-directions, $u H$ and $v H$, by displaying the ratio of the after to the before values; we also show the ratio of the corresponding local Froude numbers. An interesting feature here is the generation of significant transports, $v h$, in the transverse direction (see Figure $7 \mathrm{c}$ ), and the evidence of some unexpected resonance behaviour for a non-dimensionalized barrier gap width of 0.1 and 0.7 .

\section{Discussion}

The most striking feature of our results is the nonlinear nature of the effect of topographic barriers on reducing the tidal range at the landward side of the barrier. In all cases considered, that is, for a sub-surface barrier extending across the estuary (case (A) and (B)), or for a surface-penetrating barrier extending only partially across the estuary (case (C)), we have found that the larger the tidal forcing at the estuary mouth, the greater is the relative reduction in the tide behind the barrier. Further, our analysis shows that this is due primarily to the nonlinear bottom stress term. We have argued that for strongly nonlinear flows, when the advective terms in the momentum equations are significant, it is necessary to enhance the traditional drag coefficient in the manner described in the Appendix. However, for most of our results this enhancement is not significant, and we conclude that it is indeed the bottom stress which is responsible for the nonlinear nature of the blocking effect by topograhic barriers. Thus, one can conclude that in considering the effect of tidal barriers of the type considered here, the strength of the tide itself is an important parameter, whose effect cannot be estimated by simple linear extrapolation.

The role of the barrier geometry can be inferred from Figure 5 (case (A)), Figure 6 (case (B)) and Figure 7 (case (C)). In the case of a vertical gap (cases (A) and (B)) we see 
that the most pronounced effect is due, not unexpectedly, to the barrier depth as opposed to its along-channel width. In the case of a horizontal gap (case $(\mathrm{C})$ ), we see, again not unexpectedly, that most dramatic effects occur when the gap is quite small. Although the present results are, because of numerical necessity, restricted to topographic barriers of finite along-channel widths ( $\geq 250$ meters in these simulations), the analogous results for tidal barrages extending across the channel, of effectively zero width, can be inferred by extrapolation from the graphs shown in Figure 5 and 6 .

\section{Acknowledgements}

The author wishes to thank Professor Ron Smith for encouragement and useful discussions on the work present in this paper. I also thank Professor Roger Grimshaw for discussion, in particular for advice on the content of the appendix. Finally, this work is supported by the EPSRC of UK. 


\section{Appendix}

In order to indicate how we arrive at the expression in (2.7) for the parametrization of the short-scale waves, it is sufficient to consider just the situation when there is one horizontal spatial co-ordinate and the Coriolis term is absent. Then the full horizontal momentum equation is

$$
U_{t}+U U_{x}+W U_{z}+\frac{1}{\rho} p_{x}=\frac{\partial}{\partial z}\left(\nu(z) \frac{\partial U}{\partial z}\right),
$$

where $U$ and $W$ are the full horizontal and vertical velocity components respectively, and $\nu(z)$ is a vertical eddy viscosity coefficient. To obtain the shallow water equation variant of this, we assume that the flow is hydrostatic, so that $p_{x}=\rho g \zeta_{x}$, and then integrate over the depth to obtain

$$
\frac{\partial}{\partial t}\left\{\int_{-h}^{\zeta} U d z\right\}+\frac{\partial}{\partial x}\left\{\int_{-h}^{\zeta} U U d z\right\}+g H \zeta_{x}=\left[\nu U_{z}\right]_{-h}^{\zeta},
$$

where $H=h+\zeta$ is the total water depth. Here we have used the exact kinematic boundary condition at the free surface. Next we introduce $u$, the depth-averaged horizontal velocity, so that

$$
H u=\int_{-h}^{\zeta} U d z
$$

where $H=h+\zeta$ is the total depth. Then we let $U=u+u^{\prime}$ whereu' is now a representation of the short-scale waves. Further, we make the usual assumption that $\left(\nu U_{z}\right)_{z=-h}$ is parametrized by $C_{D}|u| u$, although for a discussion of how this may be modified by shortscale wind-waves, see Tang and Grimshaw (1997). Then we average (A.2) over the phase of the short-scale waves to obtain, at leading order with respect to $u^{\prime}$,

$$
\begin{gathered}
\qquad(H u)_{t}+(H u u)_{x}+g H \zeta_{x}=-C_{D}|u| u-D, \\
\text { where } \quad D=<\left\{\int_{-h}^{\zeta} u^{\prime} u^{\prime} d z+\left.2 U u^{\prime} \zeta^{\prime}\right|_{z=\zeta}\right\}_{x}>\text {, }
\end{gathered}
$$

where $<\cdot>$ denote the averaging operation. In the absence of any short-scale waves, i.e. $D=0,($ A.4) reduces to $(2.1)$.

The next step is to obtain a parametrisation of $D$. As discussed in the text in Section 2 , there are two approaches which have often been used. One is to use a mixing-length hypothesis, with the outcome that a traditional viscous term then appears in the horizontal momentum equations, with a horizontal eddy coefficient. Another approach, closer in spirit to that which we shall use, is to model the short-scale waves directly by retaining higher-order dispersive terms in the horizontal momentum equations, thus replacing equations (2.1-3) with a Boussinesq system. Both these options raise the order of the governing differential equations, with a consequent increase in the computational complexity of the numerical scheme.

Here, instead, we adopt a simpler approach which is based essentially on dimensional analysis. We assume that the short-scale waves are close to being linear waves, so that in order of magnitude $u^{\prime}$ sqrtg $H$ scales with $\zeta^{\prime} / H$, Then the dominant term in $D$ (i.e. the first term ), scales with $g \zeta^{\prime} \zeta^{\prime} / L$ where $L$ is the length-scale of the short-scale waves. Next we assume that $\zeta^{\prime}$ scales with $\zeta$, where $\zeta$ is now the large-scale free surface displacement 
(i.e. the variable defined in equations (2.1-3)). Our reasoning here is that the shortscale waves are produced by a local steepening of the free surface, as described by the shallow-water equations, and the resolution of such a steepening into short-scale waves may described by Korteweg-de Vries dynamics (see, for instance, Whitham (1974)). The scaling of $\zeta^{\prime}$ with respect to $\zeta$ then follows from the solution of the Korteweg-de Vries equation which describes the resolution of a discontinuity locally of size $\zeta$ into a wavetrain. Of, course, the alternative hypothesis that the short-scale waves break would lead to the afore-mentioned mixing-length approach.

Further we assume that when the steepening occurs $g \zeta$ scales with $u^{2}$, which can be deduced by using a local Bernoulli relation. It then follows that $D$ scales with $(H / L) F u^{2}$ where $F=u^{2} / g H$ is the square of the local Froude number. It remains to determine the aspect ratio $H / L$. Here we assume that the short-scale waves obey Korteweg-de Vries dynamics, and hence the Ursell number $\zeta^{\prime} L^{2} / H^{3}$ is of order unity. With the relations specified above this yields $H / L$ scaling as $F^{1 / 2}$. Finally, we infer that the generation of short-scale waves is effectively a dissipative mechanism for the depth-integrated flow, and hence we parametrize $D$ by

$$
D=\alpha F^{\frac{3}{2}}|u| u \text {. }
$$

In effect, the bottom-stress drag coefficient $C_{D}$ is enhanced by the addition of the term

$\alpha F^{\frac{3}{2}}$, as described in (2.7). Here $\alpha$ is an empirical constant, which is, of course, not determined by this dimensional analysis. We also point out that the neglected terms in $D$ produce a term similar to (A.6) but with $F^{\frac{3}{2}}$ replaced by $F^{2}$, which is a higher order term, while the inclusion of non-hydrostatic terms in the pressure would lead to an even higher-order term where $F^{\frac{3}{2}}$ is replaced by $F^{\frac{5}{2}}$.

\section{References}

Hydraulic Engineering Software VIII 2000 Water Studies, 7 ed. W.R. Blain and C.A. Brebbia, Wessex Institute of Technology, UK.

Csanady, G. T. 1984 Circulation in the Coastal Ocean, D. Reidal Publishing Company, Dordrecht, Holland, 279pp.

Falconer, R. A. And Yu, G. P. 1991 Effects of Depth, Bed Slope and Scaling on Tidal Currents and Exchange in a Laboratory Model Harbour, Proceedings of Institution of Civil Engineers, Part 2, 91, 561-576.

Gill, A.E. 1982 Atmosphere-Ocean Dynamics, Academic Press, New York, 662pp

RidDle, A. M. And LEwIS, R. E. 1998 The Effects of the Tidal Barrage on the Flow and Mixing in the Tees Estuary, in Mixing and Dispersion in Stably Stratified Flows (ed. P. A. Davies), Oxford University Press, Oxford.

Tang, Y., Grimshaw, R. 1995 A Model Analysis of the Coastally Trapped Waves Generated by Tropical Cyclones, J. Phys. Ocean. 25, 1577-1598.

Tang, Y., Grimshaw, R. 1996 Radiation Boundary Conditions in Barotropic Coastal Ocean Numerical Models, J. Comp. Phys. 123, 96-110.

Tang, Y., Grimshaw, R., Sanderson, B. and Holland, G. 1996 A Numerical 
Study of Storm Surge and Tides, with Application to the North Queensland Coast, J. Phys. Ocean. 26, 2700-2711.

Tang, Y., Holloway, P. and Grimshaw, R. 1997. A numerical study of the storm surge generated by Tropical Cyclone Jane, J. Phys. Ocean., 27, 963-976.

Whitham, G. B. 1974 Linear and Nonlinear Waves, J. Wiley, New York, 628pp.

\section{Appendix III. Notation}

The following symbols are used in this paper:

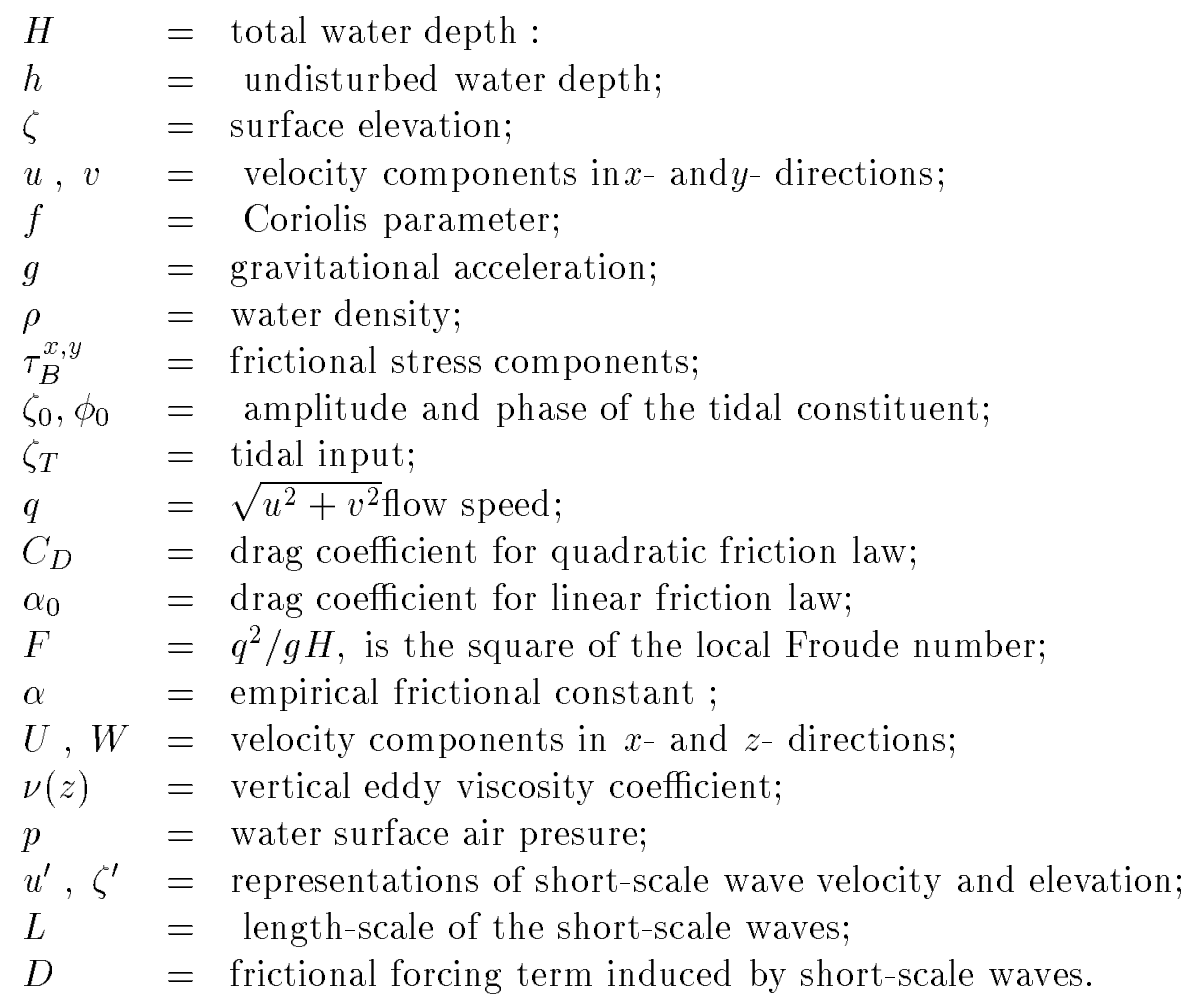




\section{Figure captions}

Figure 1 Schematic diagram of the model geometry and notation.

Figure 2 Comparison of the water level at a point before the barrier (solid lines), and a point after the barrier (dashed lines), (points B and L in Figure 1), for the full equations, including both the nonlinear advection and the nonlinear friction terms, with tidal forcing inputs $\zeta_{0}=0.1$ (first panel at the top), 0.3 (second panel) and 1.0 (third panel) meters.

Figure 3 As in Figure 2 for tidal forcing inputs of $\zeta_{0}=0.1$ (first panel at the top), and 1.0 (second panel at the bottom) meters, but the only nonlinear terms in the equations are the friction terms.

Figure 4 As in Figure 3, but with the nonlinear friction term replaced with a linear friction law.

Figure 5 Results for case (A). Ratio of peak water levels $\zeta_{A}, \zeta_{B}$ (a) and (b), and along-channel transport (c) and (d) for a point $L$ after and a point $S$ before the barrier, (see Figure 1), as a function of the barrier width $E$, for the corresponding barrier heights of 20 meters (solid lines), 17 meters (dashed lines) and 15 meters (dash dot lines); that is, the vertical gap $G=2,5$, and 7 meters respectively. Cases (a) and (c) are for an input of $\zeta_{0}=1$ meter, and (b) and (d) are for an input of $\zeta_{0}=0.5$ meters.

Figure 6 Results for case (B). As in Figure 5, but for barrier heights of 18.5, 17.5, and 16.5 meters (i.e. $G=3.5,4.5$ and 5.5 meters), and a tidal forcing input $\zeta_{0}$ of 1.0 meter.

Figure 7 Results for case (C). In (a) we show the ratio of peak water levels before and after the barrier, as a function of the non-dimensional barrier gap $G Y$, see Figure 1; in (b) we show the ratio of the after to the before values for the square of the Froude number (solid line), and the ratio of transports in the $x$ - and $y$ - directions $u H$ (dot dash) and $v h$ (dashed); in (c) we show the ratio of transports for the $x$ - and $y$-directions at a point $L$ after the barrier. Here the tidal forcing input is $\zeta_{0}=1.0$ meter. 


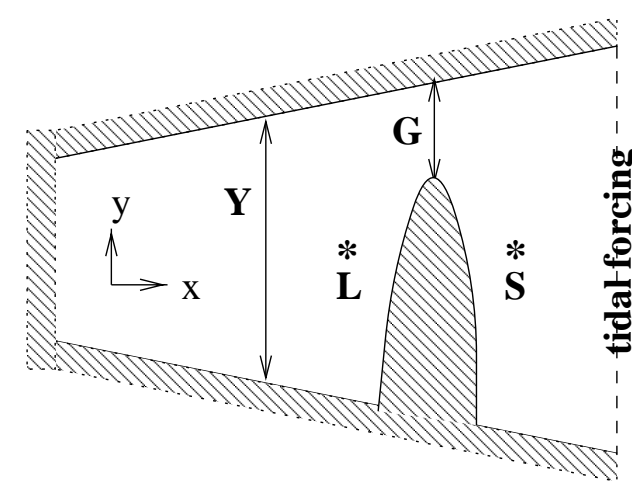

(a) Plan View

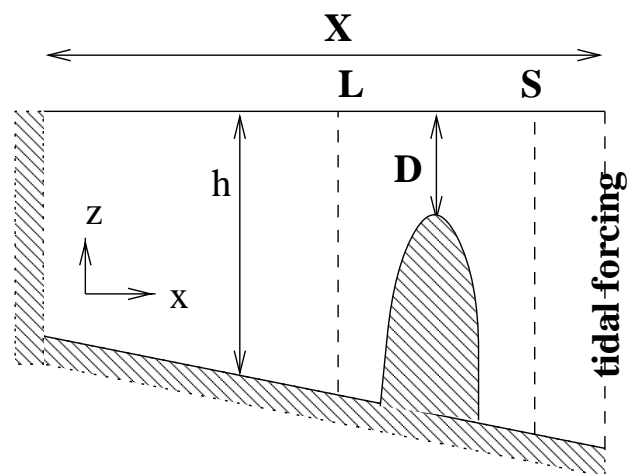

(b) Side View

Figure 1 Schematic diagram of the model geometry and notation. 

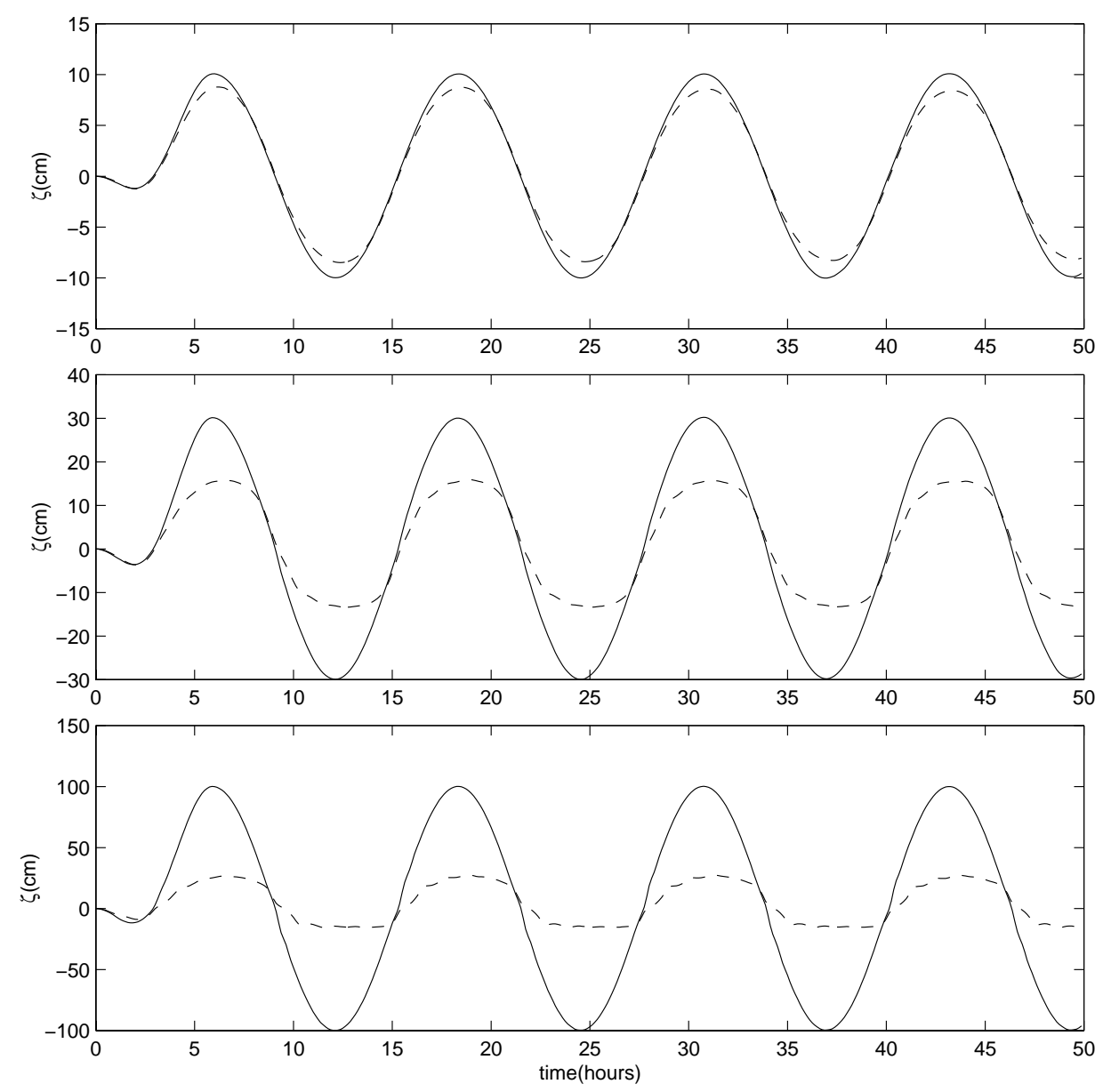

Figure 2 Comparison of the water level at a point before the barrier (solid lines), and a point after the barrier (dashed lines), (points B and L in Figure 1), for the full equations, including both the nonlinear advection and the nonlinear friction terms, with tidal forcing inputs $\zeta_{0}=0.1$ (first panel at the top), 0.3 (second panel) and 1.0 (third panel) meters. 

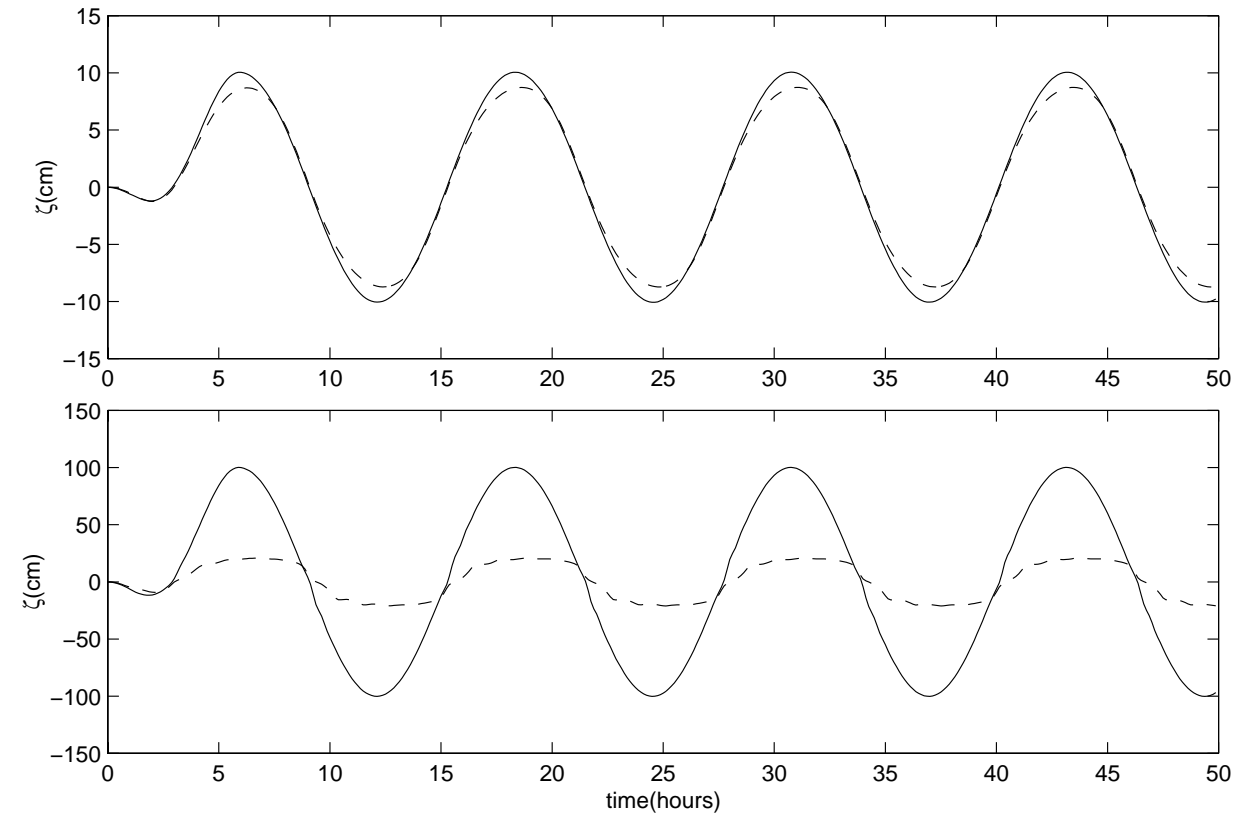

Figure 3 As in Figure 2 for tidal forcing inputs of $\zeta_{0}=0.1$ (first panel at the top), and 1.0 (second panel at the bottom) meters, but the only nonlinear terms in the equations are the friction terms. 

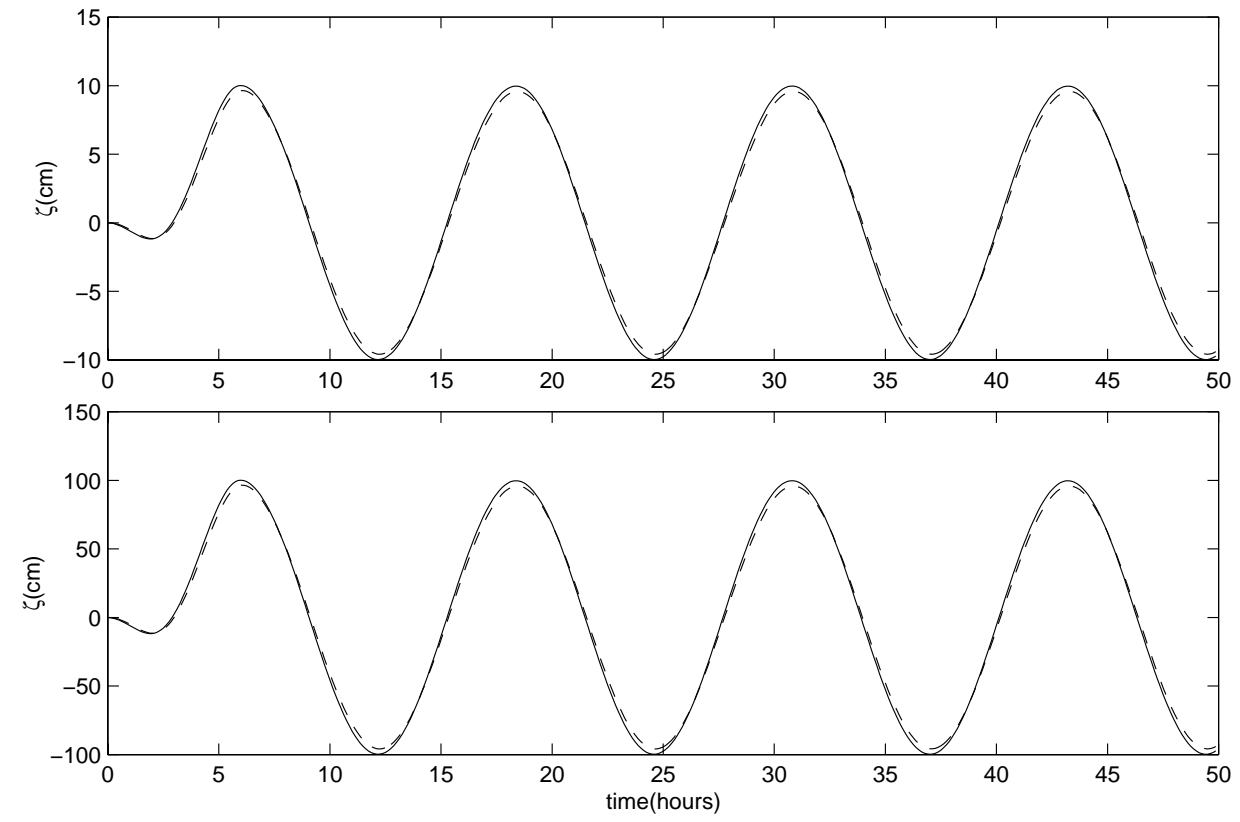

Figure 4 As in Figure 3, but with the nonlinear friction term replaced with a linear friction law. 


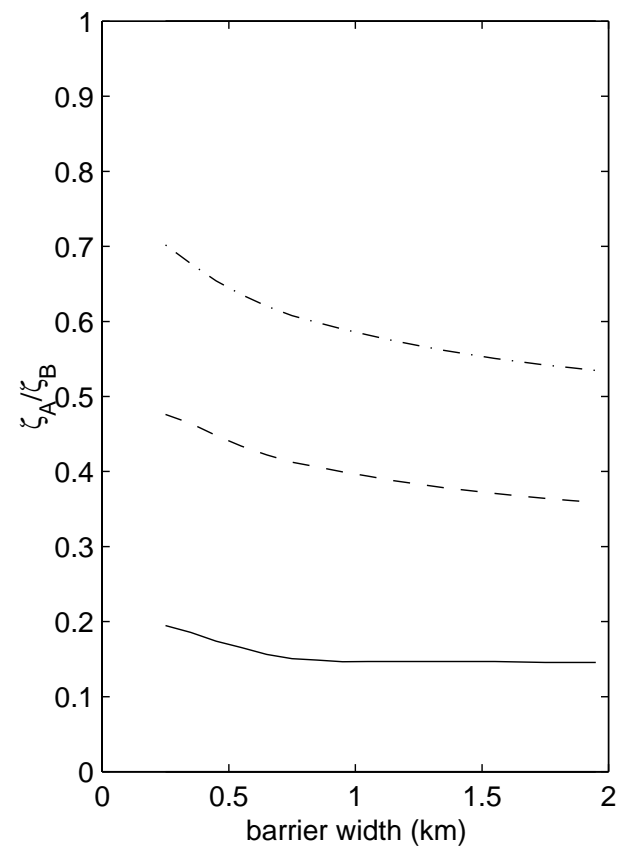

(a)

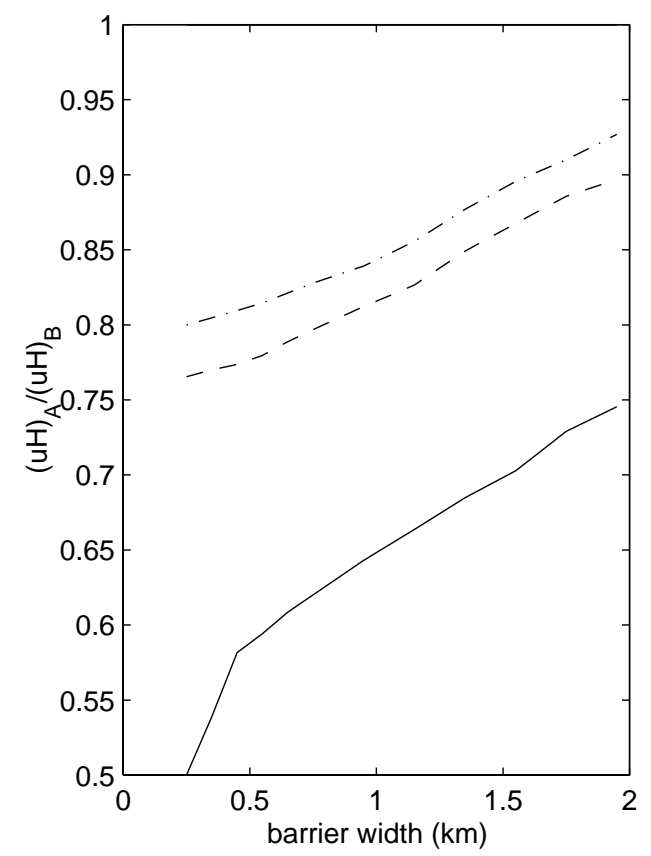

(c)

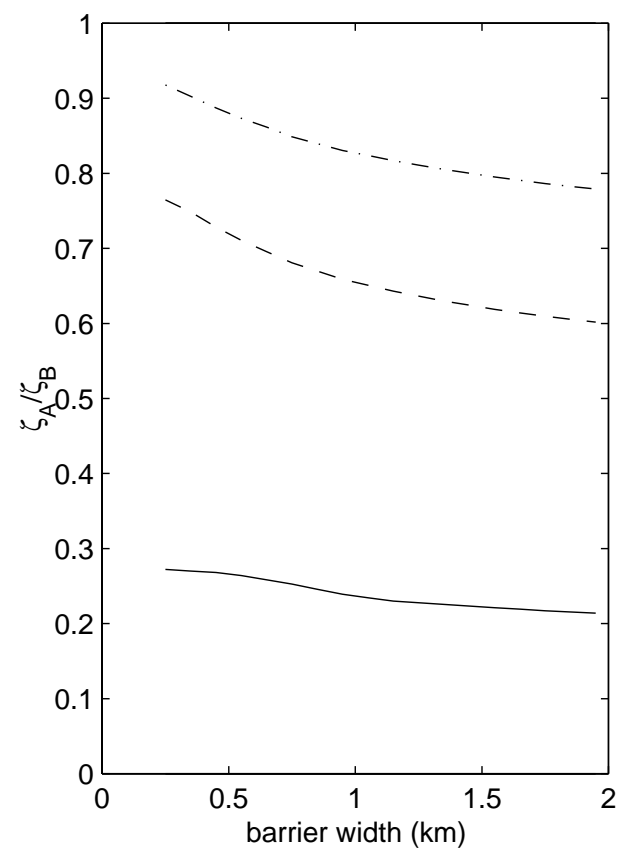

(b)

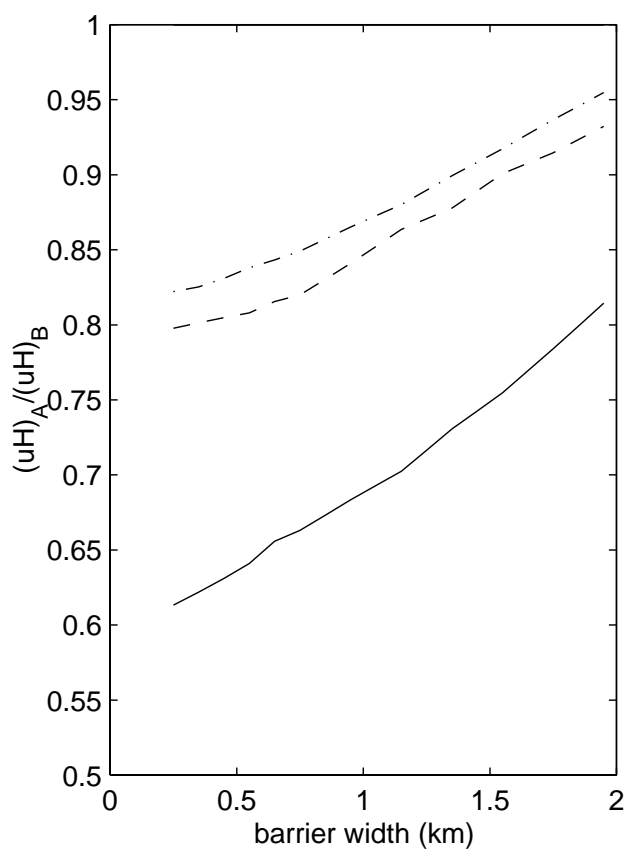

(d)

Figure 5 Results for case (A). Ratio of peak water levels $\zeta_{A}, \zeta_{B}$ (a) and (b), and along-channel transport (c) and (d) for a point $L$ after and a point $S$ before the barrier, (see Figure 1), as a function of the barrier width $E$, for the corresponding barrier heights of 20 meters (solid lines), 17 meters (dashed lines) and 15 meters (dash dot lines); that is, the vertical gap $G=2,5$, and 7 meters respectively. Cases (a) and (c) are for an input of $\zeta_{0}=1$ meter, and (b) and (d) are for an input of $\zeta_{0}=0.5$ meters. 


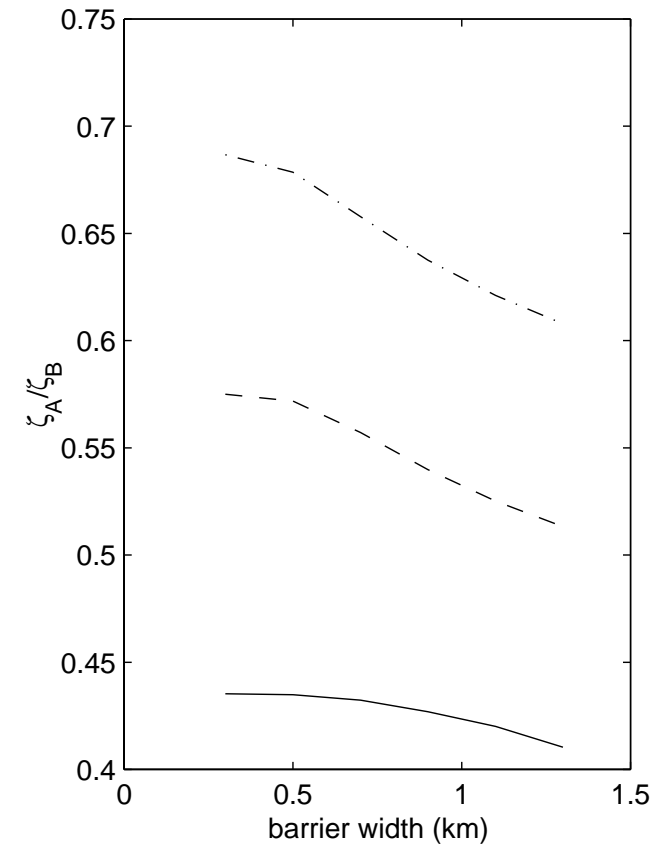

(a)

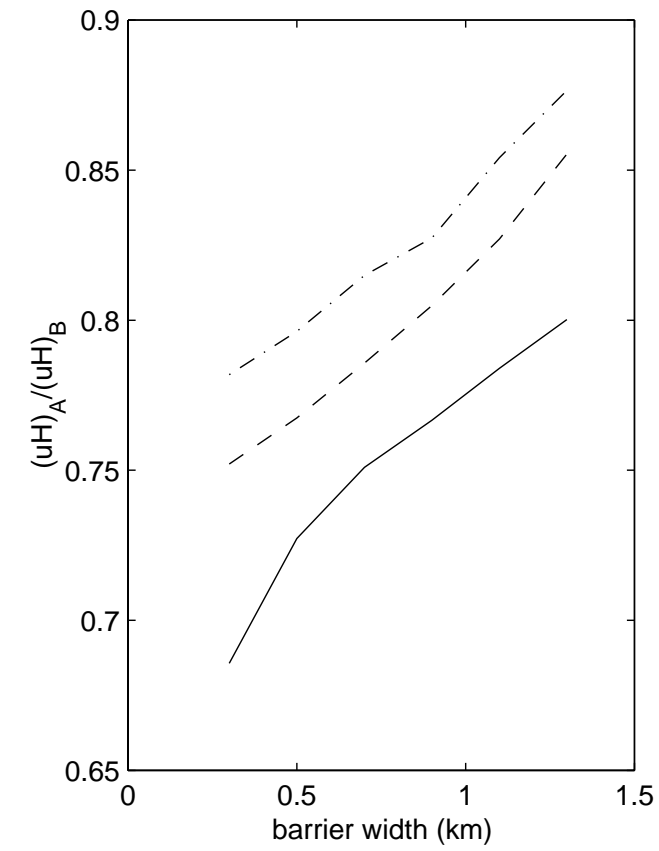

(b)

Figure 6 Results for case (B). As in Figure 5, but for barrier heights of 18.5, 17.5, and 16.5 meters (i.e. $G=3.5,4.5$ and 5.5 meters), and a tidal forcing input $\zeta_{0}$ of 1.0 meter. 


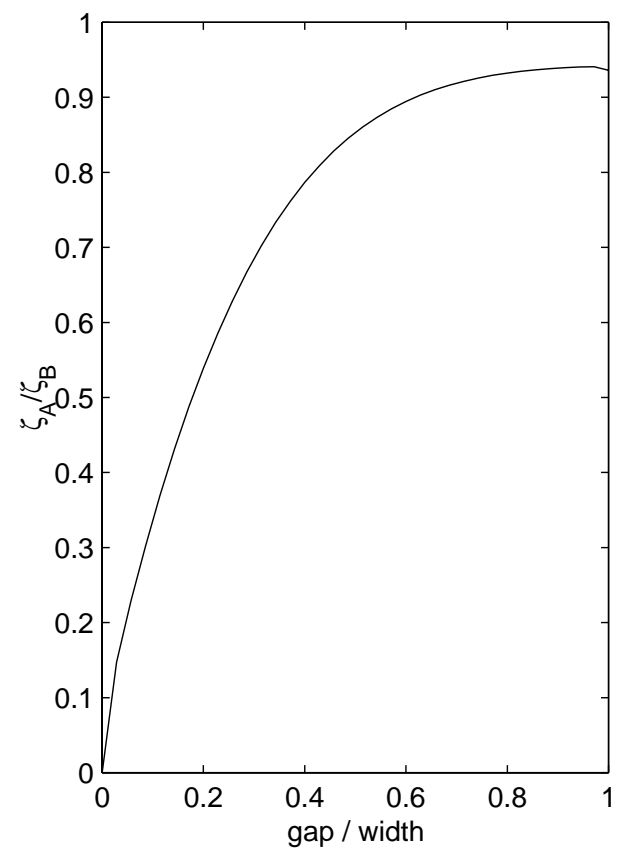

(a)

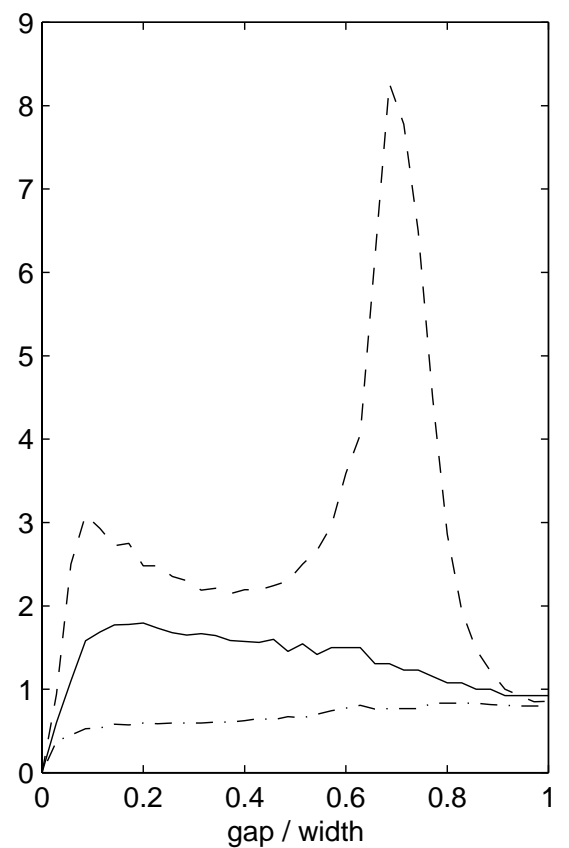

(b)

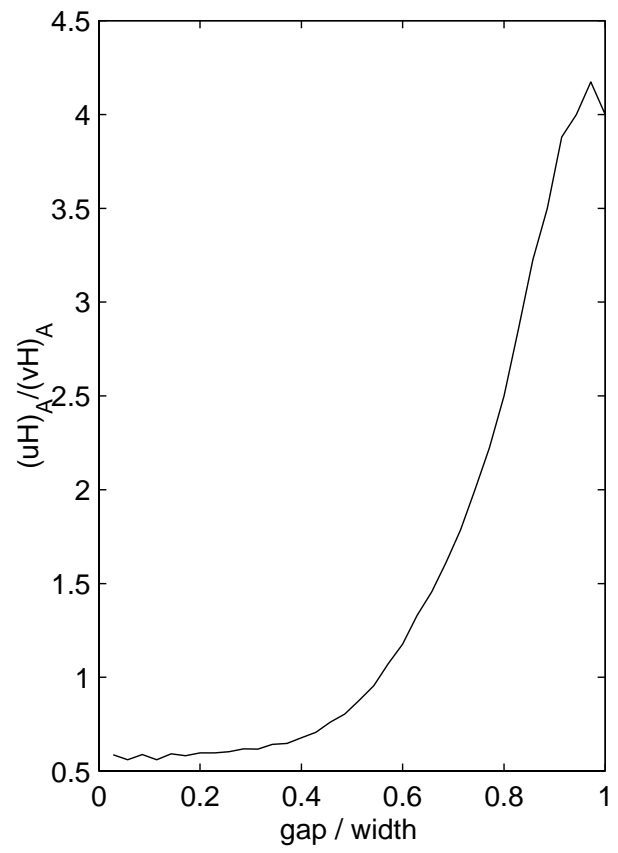

(c)

Figure 7 Results for case (C). In (a) we show the ratio of peak water levels before and after the barrier, as a function of the non-dimensional barrier gap $G Y$, see Figure 1; in (b) we show the ratio of the after to the before values for the square of the Froude number (solid line), and the ratio of transports in the $x$ - and $y$ - directions $u H$ (dot dash) and $v h$ (dashed); in (c) we show the ratio of transports for the $x$ - and $y$ - directions at a point $L$ after the barrier. Here the tidal forcing input is $\zeta_{0}=1.0$ meter. 